Meta

Journal des traducteurs

Translators' Journal

\title{
Le vocabulaire du feu
}

\section{Bruno Couture}

Volume 13, numéro 4, décembre 1968

URI : https://id.erudit.org/iderudit/002432ar

DOI : https://doi.org/10.7202/002432ar

Aller au sommaire du numéro

Éditeur(s)

Les Presses de l'Université de Montréal

ISSN

0026-0452 (imprimé)

1492-1421 (numérique)

Découvrir la revue

Citer cet article

Couture, B. (1968). Le vocabulaire du feu. Meta, 13(4), 190-192.

https://doi.org/10.7202/002432ar

Ce document est protégé par la loi sur le droit d'auteur. L'utilisation des services d'Érudit (y compris la reproduction) est assujettie à sa politique d'utilisation que vous pouvez consulter en ligne.

https://apropos.erudit.org/fr/usagers/politique-dutilisation/
Cet article est diffusé et préservé par Érudit.

Érudit est un consortium interuniversitaire sans but lucratif composé de l'Université de Montréal, l'Université Laval et l'Université du Québec à Montréal. Il a pour mission la promotion et la valorisation de la recherche. https://www.erudit.org/fr/ 


\section{LE VOCABULAIRE DU FEU}

L'ambitieux programme de recyclage mis en œuvre au Québec par les pouvoirs publics, de concert avec les collectivités locales, à l'intention des personnels techniques des services d'incendie, exige une mise au point de la terminologie française $d u$ feu, en vue de l'adapter aux réalités spécifiques de notre continent. L'entreprise présente bien certaines difficultés qu'on ne saurait sous-estimer, mais elles ne sont pas insurmontables.

D'abord, au chapitre des néologismes, signalons les termes préventionniste de l'incendie ${ }^{1}$ (fire prevention inspector) ou, plus couramment, préventionniste, souvent utilisés en France et dont la diffusion chez nous est déjà commencée. Comme ces mots sont d'excellente frappe et répondent à un réel besoin, tout porte à croire qu'ils resteront et l'on peut espérer qu'ils figureront bientôt au dictionnaire.

Dans la nomenclature des moyens hydrauliques (pumping equipment) dont disposent les corps de sapeurs-pompiers (fire brigades), il y a d'abord l'expression fire hydrant (ou fire plug) dont la traduction peut nous donner du mal. L'auteur $\mathrm{du}$ texte original tenant pour acquis que dans notre pays on n'installe que des bornes ou poteaux d'incendie dans les agglomérations, il n'y a généralement pas lieu pour lui de préciser s'il s'agit de post hydrants ou de flush hydrants (qu'on appelle respectivement en Angleterre pillar hydrants et pit ou underground hydrants). Les Européens francophones ne parlent guère que de bouches d'incendie, le climat de leurs pays leur permettant de n'installer des bornes d'incendie que dans les régions où la neige est épaisse en hiver. Néanmoins, comme on ne trouve ici de bouches d'incendie que sur les aéroports et dans certaines installations portuaires, le traducteur peut généralement, sans crainte de se tromper, rendre hydrant par borne ou poteau d'incendie. En cas de doute, on pourrait toujours, à la rigueur, employer le générique prise d'eau d'incendie, qui s'applique aussi bien au poteau qu'à la bouche.

À ce propos, $\mathrm{j}$ 'ai constaté que la réglementation et les constructeurs français de matériel d'extinction utilisent exclusivement poteau, tandis que les dictionnaires encyclopédiques donnent la première place à borne. Comme beaucoup de Canadiens français appellent fautivement cet appareil borne-fontaine, c'est sans doute le mot borne qui a les meilleures chances de s'implanter au Canada. D'autre part, sur le plan technique, il n'est pas sans intérêt de signaler qu'il existe en Amérique un type de bouche ne nécessitant pas l'utilisation de la pièce de jonction intermédiaire, généralement à robinets, qui tient lieu de poteau et que les Français appellent retenue (chuck).

Les différences entre notre matériel de première intervention (first aid equipment) et celui des Européens soulèvent également des problèmes pour le traducteur. Ainsi, il y a ce que les gens du métier appellent là-bas le secours ordinaire et qui se nomme en anglais first aid standpipe ou parfois small hose standpipe. C'est l' " ensemble de canalisations d'alimentation en eau des robinets d'incendie » (hose stations) "placés dans un établissement pour en assurer la défense contre l'incendie $\gg^{2}$. Jusqu'ici, tout va bien, mais là où les choses se gâtent, c'est lorsqu'on découvre que la contrepartie de cette insta'lation, dite grand secours - « ensemble

1. Technique du feu, vol. $6, \mathrm{n}^{0} 3$ (1965), p. 35 .

2. Colonel R. Beltramelli, Précis de prévention, Paris, France-Sélection, p. Aª 11. 
de canalisations et de déversoirs permettant d'inonder certains locaux particulièrement exposés à l'incendie ${ }^{3}-n^{\prime}$ 'existe pas chez nous. Comment parler du secours ordinaire si nous n'avons pas de grand secours? Le problème ne peut être résolu que par une étude d'ensemble des différents types d'installations fixes destinées à protéger les bâtiments contre les dangers d'incendie.

Le engins-pompes (pumping engines) présentent, eux aussi, des différences sensibles. D'abord, contrairement aux autopompes (pumpers) européennes, généralement équipées de dévidoirs fixes ou mobiles sur lesquels sont enroulés les tuyaux, la plupart de nos véhicules d'incendie comportent seulement des bacs à tuyaux (hose bodies), dans lesquels les tuyaux sont simplement pliés, en accordéon ou autrement. Aussi le sapeur qui exécute les établissements (hose lines) se nomme-t-il là-bas dévideur (hoseman), mais il est permis de se demander si cette désignation conviendrait également dans notre cas.

De même, la plupart des autopompes, en France, sont à carrosserie fermée, d'où leur nom de fourgons. Sur notre continent, dont le climat est pourtant beaucoup plus rigoureux par endroits, les voitures d'intervention sont généralement à carrosserie ouverte, de sorte qu'il faut faire, là encore, les adaptations qui s'imposent.

En ce qui concerne la manæuvre (evolutions), il existe également, d'un continent à l'autre, des différences qui revêtent une certaine importance pour le technicien, mais dont la traduction ne peut pas toujours rendre compte. Ainsi, pour ne citer qu'un cas, l'établissement normal se fait, en France, du point d'attaque vers le point d'alimentation, et l'établissement retour, inversement. Pour le sapeur américain ou canadien, le straight lay s'exécute dans le sens prévu au moment où les tuyaux ont été rangés dans le véhicule, c'est-à-dire suivant le « sexe » du demi-raccord à vis équipant l'extrémité libre du tuyau, et le reverse lay, en sens contraire, en intercalant un raccord intermédiaire (adapter) entre la prise d'eau et la ligne de tuyaux.

Un problème identique peut se poser en ce qui concerne certains matériels de sauvetage. C'est le cas, par exemple, de l'échelle à crochets des sapeurs français (en Angleterre, hook ladder) qui, suspendue à l'entablement des fenêtres, permet d'escalader les façades des bâtiments. Les pompiers américains et canadiens utilisent une échelle à peu près identique, appelée roof ladder, mais, comme son nom l'indique, elle s'accroche au faîte des toits en pente, et ne sert donc pas en position verticale.

Quant au personnel d'intervention, il comprend les volunteer firemen (qu'on pourrait appeler bénévoles), les call men (qui correspondent aux sapeurs-pompiers volontaires de France) et les permanent fire fighters (sapeurs-pompiers professionnels). À cet égard, on se gardera du faux ami sapeur-pompier permanent, car cette appellation désigne en France une fonction bien déterminée, qui n'a pas d'équivalent chez nous. En effet, les sapeurs-pompiers permanents «sont des fonctionnaires communaux, qui, en plus de leurs attributions propres, assurent le service d'incendie en restant constamment à proximité du matériel d'incendie, tant le jour (sur le lieu de leur travail) que la nuit (dans leur logement) ${ }^{4}$.

3. Grand Larousse encyclopédique.

4. Albert Faure et René Beltramelli, le Feu, Paris, Presses Universitaires de France, 1952, p. 47. 
Les différences entre l'anglais et l'américain sont nombreuses dans le domaine de la protection contre le feu, ce qui n'est pas sans compliquer les recherches lexicales. Outre le cas de post hydrant et flush hydrant, cité plus haut, mentionnons deux exemples seulement: la pénétration par effraction, appelée breaking-in en Angleterre, mais forcible entry aux États-Unis, et le seau-pompe (Br.: buckethandpump; U.S.: pump-tank), « appareil constitué par l'assemblage fixe ou amovible d'une pompe à main et d'un seau formant réserve d'eau "s ${ }^{\text {. }}$. Il en existe un modèle, le seau-pompe dorsal (back-pack ou Indian pump), qui est utilisé notamment par les groupes de défense forestière (fire suppression organizations), entre autres pour la lutte contre les feux de rémanents (slash fires).

Dans le même ordre d'idées, les nombreuses lacunes des dictionnaires anglaisfrançais, en ce qui a trait à la technique du feu, sont un autre obstacle au progrès des recherches terminologiques. Bien entendu, la chose s'explique fort bien lorsqu'il s'agit de termes désignant des dispositifs d'invention assez récente, comme les exutoires en toiture (roof vents), encore que leur ancêtre, la cheminée d'appel des théâtres, ne date pas d'hier. Ce sont des trappes dont l'ouverture est généralement assurée d'une façon automatique par la rupture d'un fusible et qui servent à l'évacuation des fumées et des gaz chauds, la concentration de ceux-ci étant parfois réalisée au moyen d'écrans verticaux (draft curtains ou curtain boards) disposés à intervalles réguliers sous la toiture.

En revanche, ce silence des dictionnaires courants est assez déconcertant dans le cas de mots utilisés depuis longtemps par les spécialistes du feu, tels tricoise (spanner), clé de barrage (hydrant key), étrangleur (hose clamp) et cordon de sécurité (fire lines).

Les flottements de l'usage, tant anglais que français, sont une cause supplémentaire d'embarras pour celui qui essaie de mettre de l'ordre dans le vocabulaire. Je citerai un cas extrême, celui de la substance qui provoque ou stabilise l'émulsion de deux liquides insolubles l'un dans l'autre ou qui peut faire mousser abondamment un liquide dans lequel elle est dissoute. Les Anglo-Saxons l'appellent indifféremment air foam stabilizer, foam compound, foam liquid, foam-forming agent (concentrate ou material), foam-making compound (material ou solution), foam stabilizing agent, foaming agent. Les Français, eux, pour n'être pas en reste, se contentent (!) d'émulsifiant, liquide émulsifiant, substance émulsifiante, émulseur, liquide émulseur, produit émulseur, moussant, agent moussant, liquide moussant, produit moussant, substance moussante, émulsionnant, agent émulsionnant: on n'a que l'embarras du choix.

Le vocabulaire $\mathrm{du}$ feu pousse d'abondantes ramifications dans les domaines de la chimie, de l'hydraulique et du bâtiment, mais je n'entends pas entrer plus avant dans cette nomenclature, qui risque de devenir vite fastidieuse pour ceux dont il ne constitue pas la sphère propre. Mon propos était simplement de signaler les travaux en cours et de donner un aperçu des difficultés auxquelles ils se heurtent, difficultés qui ne devraient cependant pas empêcher la mise au point, dans des délais raisonnables, d'une terminologie technique du feu aussi complète que possible et tenant compte des réalités nord-américaines.

Bruno COUTure

5. Grand Larousse encyclopédique. 